Yveline Lebreton · Eric Michel

\title{
Reference grids of stellar models and oscillation frequencies:
}

\section{Data from the CESAM stellar evolution code and ADIPLS oscillation programme}

Received: date / Accepted: date

\begin{abstract}
We present grids of stellar models and their associated oscillation frequencies that have been used by the CoRoT Seismology Working Group during the scientific preparation of the CoRoT mission. The stellar models have been calculated with the CESAM stellar internal structure and evolution code while the oscillation frequencies have been obtained from the CESAM models by means of the ADIPLS adiabatic oscillation programme. The grids cover a range of masses, chemical compositions and evolutionary stages corresponding to those of the CoRoT primary targets. The stellar models and oscillation frequencies are available on line through the Evolution and Seismic Tools Activity (ESTA) web site.
\end{abstract}

Keywords stars: evolution - stars: interiors · stars: oscillations $\cdot$ methods: numerical

PACS 97.10.Cv $\cdot 97.10 . \mathrm{Sj} \cdot 95.75 . \mathrm{Pq}$

\section{Introduction}

During the scientific preparation of the CoRoT mission, a reference frame in the $\mathrm{H}-\mathrm{R}$ diagram had to be provided for the CoRoT potential target stars. In particular, the critical selection of the primary targets and the census and characterisation of the stellar content in the fields surrounding the potential primary targets have required to locate all considered stars (or stellar systems) in the H-R diagram and eventually to estimate their mass, evolutionary stage and expected oscillation spectra. For that purpose we have calculated the internal structure and evolution of stars in a range of masses, chemical compositions and evolution stages of interest for

Y. Lebreton

Observatoire de Paris, GEPI, CNRS UMR 8111, 5 Place Janssen, 92195 Meudon, France

Tel.: +33-299236386

Fax: +33-299236957

E-mail: Yveline.Lebreton@obspm.fr

E. Michel

Observatoire de Paris, LESIA, CNRS UMR 8109, 5 Place Janssen, 92195 Meudon, France the modelling of CoRoT targets (Michel and Baglin, 2005). We have used the CESAM 1 stellar evolution code (Morel, 1997; Morel and Lebreton, 2007). We have selected several models along each evolution sequence and we have calculated their oscillation frequencies by means of the ADIPLS 2 programmes (Christensen-Dalsgaard, 1982, 2007).

In this paper we briefly present these grids of models and oscillation frequencies. Section 2 presents the CESAM numerical code and the input physics and initial parameters used to calculate the grids while Section 3 presents the oscillation data obtained from the ADIPLS adiabatic oscillation code. The present models and associated oscillation frequencies have been made available on the ESTA web site?.

\section{CoRoT/CESAM stellar models}

2.1 Input physics and numerical aspects

The CESAM stellar evolution code has been written and developed by P. Morel and collaborators since the late 1980's. The general description of the code is given by Morel and Lebreton (2007) but see also Morel (1997). CESAM is a public code.

The source is available on-line at the web site-http://www .obs-nice.fr/c - together with a short guide of directions for use and a comprehensive document giving a complete description of the numerics and of the physics implemented. All the models presented here were calculated in 2003 with the 4.2 version of CESAM written in Fortran77. We point out that the present version of CESAM, CESAM2k, was re-programmed in Fortran95; it has also been updated with new input physics and has been enriched with the inclusion of new physical processes (Morel and Lebreton, 2007).

The models have be initialised on the zero age main sequence (ZAMS) and the evolution has been followed up to

1 Code d'Evolution Stellaire Adaptatif et Modulaire

2 Aarhus Adiabatic Pulsation Package), available at http://astro.phys.au.dk/jcd/adipack.n

3 http://www.astro.up.pt/corot/models/ and also on the Paris Observatory web site at http://wwwusr.obspm.fr/ lebreton/Modeles/CESAM_COROT.html 
the beginning of the red giant branch. The models have typically 600 mesh points in the interior while the atmosphere is restored on a grid of typically 100 grid points. During hydrogen burning the time step is adjusted according to the relative changes of the hydrogen abundances. In the present calculations it takes from 165 to 420 time steps to evolve from the ZAMS to the ascent of the red giant branch, depending on the mass.

All the models have been calculated with the same given set of standard input physics. Here, we have neglected microscopic and turbulent diffusion processes as well as rotation or magnetic fields. The input physics are the following:

- Equation of State. We used the so-called CEFF analytical equation of state (Christensen-Dalsgaard and Däppen, 1992) which is an improved version of the EFF (Eggleton. Faulkner, $c^{-2.0-}$ 1973) equation of state including the Coulomb corrections to pressure in the so-called Debye-Hückel approximation.

- Opacities. We used the OPAL95 opacity tables (Iglesias and Ri 1996) complemented at low temperatures $\left(T \lesssim 10^{4} \mathrm{~K}\right)$ by the Alexander and Ferguson (1994) tables. The metal mixture of the opacity tables is fixed to the initial mixture of the model (see below).

- Nuclear reaction rates. We used the basic pp and CNO reaction networks up to the ${ }^{17} O(p, \alpha){ }^{14} N$ reaction. In the present models the CESAM code takes ${ }^{7} \mathrm{Li},{ }^{7} \mathrm{Be}$ and ${ }^{2} \mathrm{H}$ at equilibrium. The nuclear reaction rates are from Caughlan and Fowler (1988). Weak screening is assumed under Salpeter's formulation (1954).

- Convection and overshooting. We use the classical mixing length treatment of Böhm-Vitense (1958) under the formulation of Henyey, Vardya, and Bodenheimer (1965) taking into account the optical thickness of the convective bubble. To find the location of the onset of convection, we used Schwarzschild's criterion. In models calculated with overshooting, the convective core is extended on a distance $l_{\mathrm{ov}}=\alpha_{\mathrm{ov}} \times \min \left(H_{\mathrm{p}}, R_{\mathrm{cc}}\right)$ where $\alpha_{\mathrm{ov}}$ is the overshooting parameter, $H_{\mathrm{p}}$ the pressure scale height and $R_{\mathrm{cc}}$ the radius of the convective core. The core is mixed in the region corresponding to both the convective and overshooting regions and in the overshooting region the temperature gradient is taken to be equal to the adiabatic gradient.

- Atmosphere. Eddington's grey $T(\tau)$ law is used for the atmosphere calculation. The hydrostatic equation is integrated in the atmosphere, starting at the optical depth $\tau=10^{-4}$ and the connection with the envelope is made at $\tau=10$ to ensure the validity of the diffusion approximation (Morel et al., 1994). At this level, we insure the continuity of the variables and of their first derivatives with space. The radius of the star is taken to be the bolometric radius, i.e. the radius at the level where the local temperature equals the effective temperature $(\tau=2 / 3$ for the Eddington's law).

- Initial abundances of the elements and heavy elements mixture. All models were calculated with the classical GN93 solar mixture of heavy elements from Grevesse and
(1993) corresponding to a solar metals to hydrogen ratio $(Z / X)_{\odot}=0.0245$ where $Z$ and $X$ are the abundances in mass fraction of heavy elements and hydrogen respectively. In the nuclear reaction network the initial abundance of each chemical species is split between its isotopes according to the isotopic ratio of nuclides. The initial amount of ${ }^{2} \mathrm{H}$ is assumed to be converted in ${ }^{3} \mathrm{He}$.

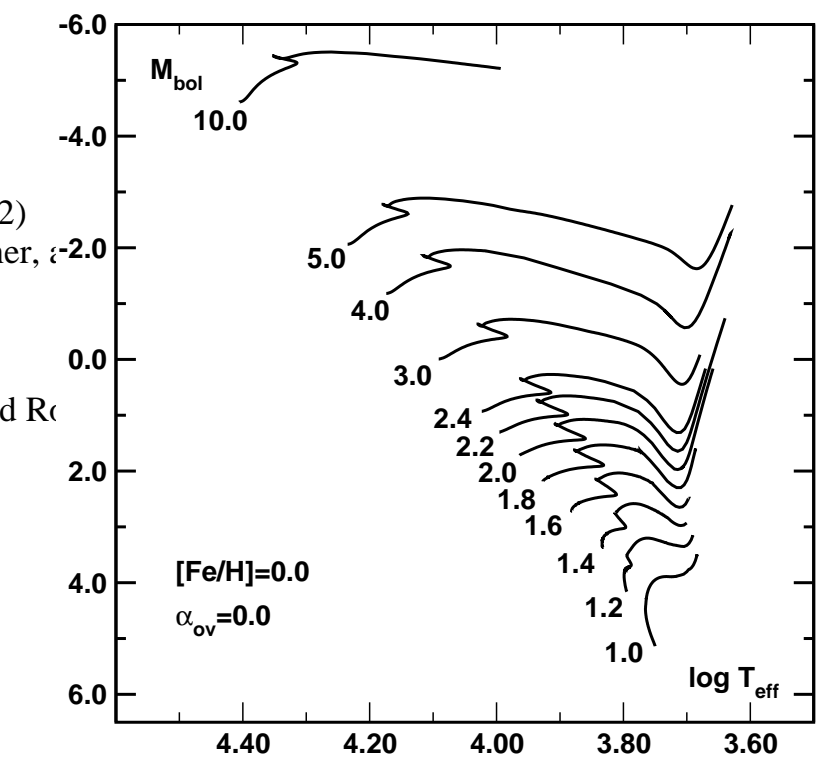

Fig. 1 Grid G1: evolutionary sequences in the H-R diagram for models with solar metallicity $[\mathrm{Fe} / \mathrm{H}]=0.0$ corresponding to $Z / X=0.0245$, helium abundance $Y=0.2672$, no overshooting.

\subsection{Initial parameters of the models}

The calibration of a solar model in luminosity and radius with the input physics given above leads to an initial abundance of helium $Y_{\odot}=0.2672$, and to a solar mixing-length parameter $\alpha_{\mathrm{MLT}, \odot}=1.63$. The corresponding initial hydrogen and metal abundances are respectively $X_{\odot}=0.7153$ and $Z_{\odot}=0.0175$.

We have calculated models for two values of the $[\mathrm{Fe} / \mathrm{H}]$ ratio: $[\mathrm{Fe} / \mathrm{H}]=0.0$ (solar) and -0.1 (metal deficient). The metallicity of the models $Z$ has been obtained from the relation $[\mathrm{Fe} / \mathrm{H}]=\log (Z / X)-\log (Z / X)_{\odot}$. The individual abundances of metals have been scaled on the solar ones.

For models with non solar metallicity, we have derived the helium abundance in mass fraction $Y$ according to the relation $Y=Y_{\mathrm{p}}+Z(\Delta Y / \Delta Z)$, where $\Delta Y / \Delta Z$ is the helium to metal enrichment ratio and $Y_{\mathrm{p}}$ is the primordial helium abundance. We took $\Delta Y / \Delta Z=\left(Y_{\odot}-Y_{\mathrm{p}}\right) / Z_{\odot} \sim 1.3$ and $Y_{\mathrm{p}}=$ 0.245 (see Olive and Skillman, 2004).

All the models were calculated with a mixing-length parameter equal to the solar one. Finally, for solar metallicity we calculated two grids of models, one without overshooting Nomedsone including overshooting with the value $\alpha_{\mathrm{ov}}=0.15$. 
We have calculated models with masses in the range covered by the CoRoT targets: 1.0, 1.2, 1.4, 1.6, 1.8, 2.0, 2.2, 2.4, 3.0, 4.0, 5.0 and $10.0 M_{\odot}$.

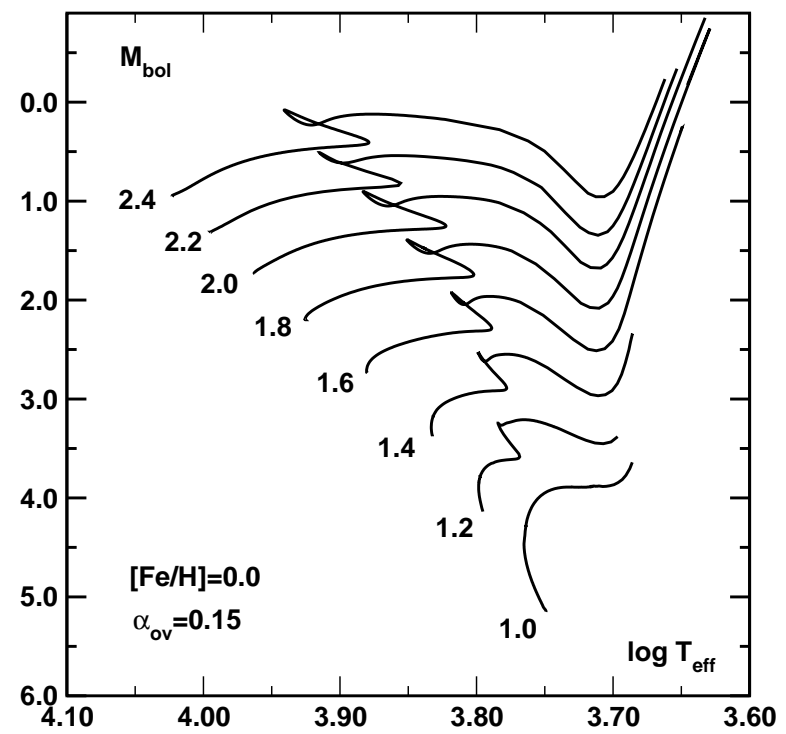

Fig. 2 Grid G2: evolutionary sequences in the H-R diagram for models with solar metallicity $[\mathrm{Fe} / \mathrm{H}]=0.0$ corresponding to $Z / X=0.0245$, helium abundance $Y=0.2672$ and overshooting $\alpha_{\mathrm{ov}}=0.15$

\subsection{Model grids}

We present three grids of models. Grid G1 consists in evolutionary sequences for the 12 values of the stellar mass listed above (between 1.0 and $10.0 M_{\odot}$ ), a solar metallicity and no overshooting. This grid covers the domain of mass of main CoRoT targets, including solar-like oscillators and $\delta$ Scuti and $\beta$ Cephei pulsators. In addition to this standard grid, we have calculated two grids restricted to solar-type and $\delta$ Scuti stars, i.e. for the 8 lower values of masses listed above, between 1.0 and $2.4 M_{\odot}$. Grid G2 consists in evolutionary sequences with solar metallicity and overshooting $\left(\alpha_{\mathrm{ov}}=0.15\right)$ and grid $\mathbf{G 3}$ consists in evolutionary sequences with $[\mathrm{Fe} / \mathrm{H}]=-0.1$ and no overshooting. Figures 1,2 and 3 display the evolutionary sequences in the $\mathrm{H}-\mathrm{R}$ diagram for the 3 grids of CESAM models available on the ESTA web site 4 . For each evolutionary sequence, we also provide on the web site a file in which the properties of the models are displayed: mass, logarithm of the effective temperature $\log T_{\text {eff }}$, bolometric magnitude, logarithm of the luminosity $\log \left(L / L_{\odot}\right)$, logarithm of the surface gravity $\log g$ and age.

\section{ADIPLS oscillation frequencies}

Using the ADIPLS stellar oscillation program, we have calculated the oscillation frequencies for a selection of models

\footnotetext{
4 http://www.astro.up.pt/corot/models/
}

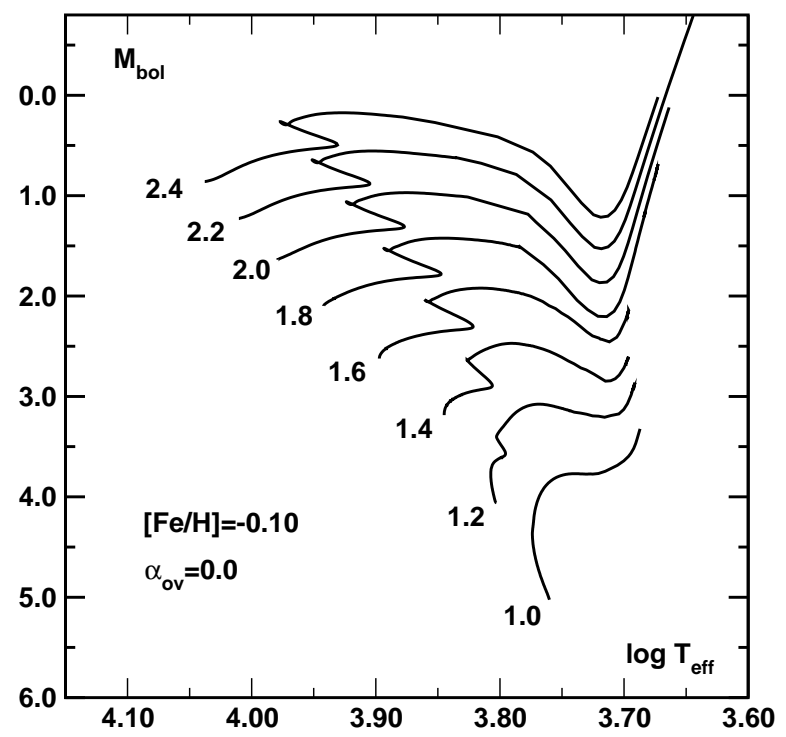

Fig. 3 Grid G3: evolutionary sequences in the H-R diagram for models with sub-solar metallicity $[\mathrm{Fe} / \mathrm{H}]=-0.1$ corresponding to $Z / X=$ 0.0194 , helium abundance $Y=0.2632$ and no overshooting

of the $\mathbf{G 2}$ grid (i.e. the grid corresponding to models with solar metallicity $[\mathrm{Fe} / \mathrm{H}]=0.0, Z / X=0.0245$, helium abundance $Y=0.2672$ and overshooting $\alpha_{\mathrm{ov}}=0.15$ ). In relation with CoRoT targets, we have chosen two values of the mass in the G2 grid: $M=1.2 M_{\odot}$ which is representative of solar like oscillators and $M=1.8 M_{\odot}$ which is representative of $\delta$ Scuti pulsators. For the $M=1.2 M_{\odot}$ sequence, we have selected models along the evolutionary track by steps of $100 \mathrm{Myr}$, except on the hook at the end of the mainsequence where steps are of $10 \mathrm{Myr}$. For the $M=1.8 M_{\odot}$ sequence, we have selected models by steps of $50 \mathrm{Myr}$ on the main-sequence and by steps of $10 \mathrm{Myr}$ from the red point to the bottom of the red giant branch.

For each mass we provide two archives on the ESTA web site, a $\mathbf{O i}$ and a $\mathbf{F i}$ archive, where $\mathrm{i}=1$ and 2 for $M=1.2$ and $M=1.8 M_{\odot}$ respectively.

The Oi archive contains ASCII files, with a .osc extension, each corresponding to a selected model on the evolutionary sequence. The .osc files can be read by means of the programme tools available in the ADIPLS package and described on the ESTA web site5. They list the global properties of the considered model (name, mass, age, initial composition, luminosity, photospheric radius, etc.) as well as a wide range of model variables at each mesh point (distance $r$ to the centre, mass inside the sphere of radius $r$, pressure, density, temperature, chemical composition, opacity and several other physical parameters including quantities of interest for the computation of adiabatic oscillations like the Brunt-Väisälä frequency).

The Fi archive contains ASCII files, with a freq extension, each corresponding to a selected model on the evolutionary sequence. The freq files are the result of the frequency calculation by the ADIPLS program. They list the

\footnotetext{
5 at http://www.astro.up.pt/corot/ntools/modconv
} 
mass, radius central pressure and density of the model and the frequency of oscillation, for each mode identified by its degree $\ell$ and order $n$. We point out that these files are not corrected for problems of numbering of the modes that occur for modes of $\ell=1$ in the region of the fundamental.

\section{Conclusion}

On the ESTA web site we have provided grids of stellar models and oscillation frequencies that have been used by the CoRoT Seismology Working Group during the scientific preparation of the CoRoT mission. The stellar models are provided for a range of masses between 1.0 and $10.0 M_{\odot}$ and chemical compositions $[\mathrm{Fe} / \mathrm{H}]=0.0$ and -1.0 dex representative of the main CoRoT targets (solar-type stars, $\delta$ Scuti stars and $\beta$ Cephei stars). Oscillation frequencies are provided all along the main-sequence track for a solar-type oscillator $(M=1.2$ $\left.M_{\odot}\right)$ and a $\delta$ Scuti pulsator $\left(M=1.8 M_{\odot}\right)$. Up to now these reference grids have been used to locate CoRoT potential targets in the $\mathrm{H}-\mathrm{R}$ diagram in the process of target selection (see Fig. 1 in Lebreton et al., 2007a) and to study some $\delta$ Scuti candidates for CoRoT (Poretti et al., 2005).

On the ESTA web site, other grids of stellar models and oscillation frequencies can be found. These stellar models have been calculated either with the CESAM or the CLES code and the oscillation frequencies have been calculated either with the ADIPLS, POSC or LOSC code. All this material (codes and grids) is described in this volume by Lebreton et al. (2007a); Montalbán et al. (2007a); Marques et al. (2007). We point out that the thorough comparisons of the stellar evolutionary codes and oscillations codes performed under ESTA have shown very good agreement between models calculated by the CESAM and CLES codes which gives us confidence in the use of the present grids (see Lebreton et al., 2007b; Montalbán et al., 2007b; Moya et al., 2007).

\section{References}

Alexander, D.R., Ferguson, J.W.: Low-temperature Rosseland opacities. ApJ 437, 879-891 (1994). doi:10.1086/175039

Böhm-Vitense, E.: Über die Wasserstoffkonvektionszone in Sternen verschiedener Effektivtemperaturen und Leuchtkräfte. Mit 5 Textabbildungen. Zeitschrift Astrophysics 46, 108+ (1958)

Caughlan, G.R., Fowler, W.A.: Thermonuclear Reaction Rates V. Atomic Data and Nuclear Data Tables 40, 283-+ (1988)

Christensen-Dalsgaard, J.: On solar models and their periods of oscillation. MNRAS 199, 735-761 (1982)

Christensen-Dalsgaard J: ADIPLS: Aarhus Adiabatic Pulsation Package. In: Astrophys. Space Sci. (CoRoT/ESTA Volume). (2007)

Christensen-Dalsgaard, J., Däppen, W.: Solar oscillations and the equation of state. A\&A Rev. 4, 267-361 (1992)
Eggleton PP, Faulkner J, Flannery BP: An Approximate Equation of State for Stellar Material. 23, 325+ (1973)

Grevesse N, Noels A: Cosmic abundances of the Elements. In: Origin and Evolution of the Elements. (1993)

Henyey, L., Vardya, M.S., Bodenheimer, P.: Studies in Stellar Evolution. III. The Calculation of Model Envelopes. ApJ 142, 841-+ (1965)

Iglesias, C.A., Rogers, F.J.: Updated Opal Opacities. ApJ 464, 943+ (1996)

Lebreton, Y., Monteiro, M.J.P.F.G., Montalbán, J., et al.: The CoRoT evolution and seismic tool activity. In: Astrophys. Space Sci. (CoRoT/ESTA Volume). (2007a)

Lebreton, Y., Montalbán, J., Christensen-Dalsgaard, J., Roxburgh, I., Weiss, A.: CoRoT/ESTA - TASK 1 and TASK 3 comparison of the internal structure and seismic properties of representative stellar models. Astrophys. Space Sci. (CoRoT/ESTA Volume) (2007b)

Michel E, Baglin A: Space and ground-based seismology. In: Casoli, F., Contini, T., Hameury, J.M., Pagani, L. (eds.) SF2A-2005: Semaine de 1'Astrophysique Francaise. pp. 283-+. (December 2005)

Montalbán, J., Miglio A., Noels A., Scuflaire R.: Grids of stellar models and frequencies with CLES + LOSC. Astrophys. Space Sci. (CoRoT/ESTA Volume) (2007a)

Montalbán, J., Lebreton, Y., Miglio A., Scuflaire R., Morel P., Noels, A.: Thorough analysis of input physics in CESAM and CLÉS. Astrophys. Space Sci. (CoRoT/ESTA Volume) (2007b)

Marques, J.P. Monteiro, M.J.P.F.G., Fernandes, J.: A CESAM+POSC grid of stellar models from the birhline to the sub-giant branch. Astrophys. Space Sci. (CoRoT/ESTA Volume) (2007)

Morel, P.: CESAM: A code for stellar evolution calculations. A\&AS 124, 597-614 (1997)

Morel P, Lebreton Y: CESAM: Code d'Evolution Stellaire Adaptatif et Modulaire. In: Astrophys. Space Sci. (CoRoT/ESTA Volume). (2007)

Morel, P., van't Veer, C., Provost, J., Berthomieu, G., Castelli, F., Cayrel, R., Goupil, M.J., Lebreton, Y.: Incorporating the atmosphere in stellar structure models: the solar case. A\&A 286, 91-102 (1994)

Moya, A., Christensen-Dalsgaard, J., Charpinet, S., et al.: Inter-comparison of the $\mathrm{g}$-, $\mathrm{f}$ - and $\mathrm{p}$-modes calculated using different oscillation codes for a given stellar model. Astrophys. Space Sci. (CoRoT/ESTA Volume) (2007)

Olive, K.A., Skillman, E.D.: A Realistic Determination of the Error on the Primordial Helium Abundance: Steps toward Nonparametric Nebular Helium Abundances. ApJ 617, 29-49 (2004). doi:10.1086/425170

Poretti, E., Alonso, R., Amado, P.J., et al.: Preparing the COROT Space Mission: New Variable Stars in the Galactic Anticenter Direction. AJ 129, 2461 (2005)

Salpeter, E.E.: Electrons Screening and Thermonuclear Reactions. Australian Journal of Physics 7, 373-+ (1954) 